\title{
Does semantic information need to be truthful?
}

\author{
Björn Lundgren 1
}

Received: 18 October 2016 / Accepted: 5 October 2017 / Published online: 24 October 2017

(C) The Author(s) 2017. This article is an open access publication

\begin{abstract}
The concept of information has well-known difficulties. Among the many issues that have been discussed is the alethic nature of a semantic conception of information. Floridi (Minds Mach 14(2):197-222, 2004; Philos Phenomenol Res 70:351-370, 2005; EUJAP 3(1):31-41, 2007; The philosophy of information, Oxford University Press, Oxford, 2011) argued that semantic information must be truthful. In this article, arguments will be presented in favor of an alethically neutral conception of semantic information and it will be shown that such a conception can withstand Floridi's criticism. In particular, it is argued that an alethically neutral conception of semantic information can manage the so-called Bar-Hillel Carnap paradox, according to which contradictions have maximum informational content. This issue, as well as some of Floridi's other arguments, is resolved by disentangling the property of being information from the property of being informative. The essay's final conclusion is that although semantic information is alethically neutral, a veridical conception of semantic information can, and should, be retained as a subconcept of semantic information (i.e., as veridical semantic information), as it is essential for the analysis of informativity, which, unlike the property of being information, depends on truth.
\end{abstract}

Keywords Philosophy of information - Semantic information - Veridicality thesis · Informativity $\cdot$ Bar-Hillel Carnap paradox $\cdot$ Information liar paradox $\cdot$ Floridi

\footnotetext{
$凶$ Björn Lundgren bjorn.lundgren@abe.kth.se

1 Division of Philosophy, Royal Institute of Technology (KTH), Brinellvägen 32, 10044 Stockholm, Sweden
} 


\section{Introduction}

Ever since Luciano Floridi re-invigorated the veridicality thesis (that [semantic] information must be true, or truthful), the discussion of this issue has been expanding (see Floridi 2004, 2005; cf. Fetzer 2004; Dodig-Crnkovic 2005). Although Floridi claims that various critical comments have "been proved unjustified, and as a result, there is now a growing consensus" about his approach (Floridi 2012, p. 432, footnotes removed), ${ }^{1}$ the discussion has continued. Recently, I argued that Floridi's proposed definitions suffer from counter-examples such that the sentence $x$ is information if, and only if, $x$ is not information (see Lundgren 2015a). The same idea was later developed and expanded by Macaulay Ferguson (2015), who furthermore argues that the choice of the definition of semantic information (between a veridical and an alethically neutral conception) is a dilemma because it is a choice between two paradoxes: information liar paradoxes and the Bar-Hillel Carnap paradox (BCP); both will be explained in this paper. This dilemma will serve as part of the dialectics of this essay.

The main aim of this essay is to argue for an alethically neutral conception of semantic information. This argument will be made by presenting counter-arguments against Floridi's main arguments for the veridicality thesis, as well as showing that a veridical conception of semantic information leads to a contradiction. I consider Floridi's arguments because he is currently the most influential proponent of the veridicality thesis and of a semantic conception of information. The main contribution of this essay is that an alethically neutral conception of semantic information can avoid the BCP, thus resolving the supposed dilemma between alethically neutral and veridical conceptions of semantic information. This is done by introducing a distinction between the property of being information and the property of being informative. Overall, combined with the other arguments, this speaks in favor of an alethically neutral conception of semantic information and against the veridicality thesis.

However, a preference for an alethically neutral conception over a veridical conception of semantic information does not mean that we cannot, or should not, retain the latter concept. I conclude that we should retain it as a subconcept of the former concept, i.e., as veridical semantic information.

The rest of the essay is structured as follows. I present the relevant background in Sect. 2. Then I present one conceptual problem for the veridicality thesis in Sect. 3. In Sect. 4, I present Floridi's first argument for the veridicality thesis and arguments against it. Finally, I end by explaining and resolving the BCP, and the dilemma, as well as the other main parts of Floridi's 'semantic argument' in Sect. 5. Before ending, I summarize my conclusions in Sect. 6. After the conclusions, an "Appendix" includes further discussion of the topic in Sect. 3.

Although the dialectic structure of this article is centered on Floridi's arguments, the arguments are not aimed only at his arguments but at a broader argumentation that he exemplifies.

In the remainder of this essay, I sometimes use 'semantic information' instead of a 'semantic conception of information', and on occasion (when what is intended

${ }^{1}$ Floridi lists only a few early critiques; he mentions Fetzer (2004), Colburn (2000a, b), and Dodig-Crnkovic (2005). 
is clearly indicated by the context), I also simply use 'information' for 'semantic information'. On occasion, I also use VT as an abbreviation for 'the veridicality thesis' and AN for 'an alethically neutral conception of semantic information'.

\section{Background and specification}

The notion of semantic information was originally introduced by Bar-Hillel and Carnap (1964[1952], cf. Bar-Hillel and Carnap 1953), and it is part of a larger context of theories of information (see Adriaans 2013 for a brief overview). Bar-Hillel and Carnap created their approach in response to contemporary theories of information, namely, the 'Mathematical Theory of Communication', or 'Theory of (Transmission of) Information', which-according to Bar-Hillel and Carnap- "authoritative presentations" have taken "great care [...] to point out that this theory is not interested in the semantic aspects of communication" (Bar-Hillel and Carnap 1964, p. 222). BarHillel and Carnap refer to two representative sources for this position: C. E. Shannon, for whom semantics issues were considered "irrelevant to the engineering problem" (Shannon quoted in ibid), and E. Colin Cherry, who thought that "semantics lies outside the scope of mathematical information theory" (Colin Cherry quoted in ibid). Bar-Hillel and Carnap focused mainly on measurements of informativity (i.e., how informative some information is).

After Bar-Hillel and Carnap, and before Floridi re-invigorated the debate on the veridicality thesis, Dretske (1981) argued, while recognizing that information "may be a semantic concept" (p. 42) that "information embodied in a signal (linguistic or otherwise) is only incidentally related to the meaning (if any) of that signal" (p. 44), and that:

[S]ignals may have a meaning but they carry information. What information a signal carries is what it is capable of "telling" us, telling us truly [...] information is that commodity capable of yielding knowledge, and [...] [i]f everything I say to you is false, then I have given you no information. (ibid)

Tellingly, he focused his analysis of information on informativity or the "amount of information" (ibid). Concerning the veridicality thesis, he famously exemplified his position by comparing "false information and mis-information" with "decoy ducks and rubber ducks" (ibid, p. 45).

Fox (1983, p. 193) took another position in relation to the verdicality thesis, arguing that information can be true or false, while misinformation is always false. His conceptual analysis focused on the relation between $x$ informs $y$ that $p$ and information, and $x$ misinforms $y$ that $p$ and misinformation, arguing that $\mathrm{x}$ informs $\mathrm{y}$ that $\mathrm{p}$ if $\mathrm{x}$ tells $\mathrm{y}$ that $\mathrm{p}$ and $\mathrm{x}$ is in a position to know that $\mathrm{p}$, and $\mathrm{x}$ misinforms $\mathrm{y}$ that $\mathrm{p}$ if $\mathrm{x}$ informs $\mathrm{y}$ that $\mathrm{p}$ and $\mathrm{p}$ is false (ibid, p. 184).

Grice in Studies in the Way of Words stated that false information "is just not information" (1989, p. 371). Barwise and Seligman (1997) developed their account mainly based on Dretske (1981) and thus in line with his view on veridicality. Graham (1999) took a position in favor of the veridicality thesis. His conceptual analysis of information can be summed up by his claim that "in normal speech 'information' is 
an epistemologically normative term: to be newly possessed of information implies that we now know something we did not know before" (p. 89).

When Floridi re-uses the concept of 'semantic information', he focuses on an epistemic sense of the concept. ${ }^{2}$ He states that he "will analyse only one crucial aspect of a specific kind of information, namely the alethic nature of declarative, semantic information, the kind of information that we normally take to be essential for epistemic purposes" (Floridi 2011, p. 82). Let us call this Floridi's 'characterization' of semantic information. Although this is certainly a specific kind of information worthy of philosophical consideration, it is prima facie an open question if there is a special kind of information that is essential for epistemic purposes, and if so, how it should be defined. I will concern myself with the latter question, i.e., which conception of semantic information — alethically neutral or veridical — fits best with Floridi's characterization of the concept of semantic information as information which is declarative, semantic, and essential for epistemic purposes. ${ }^{3}$

Floridi (2011) identifies the basic components of semantic information as data ${ }^{4}+$ meaning. ${ }^{5}$ From this, he extrapolates a more rigid definition:

GDI $\sigma$ (an infon) is an instance of semantic information if and only if:

GDI. $1 \sigma$ consists of $n$ data $(\mathrm{d})$, for $n \geq 1$;

GDI.2 the data are well-formed (wfd);

GDI.3 the wfd are meaningful $($ mwfd $=\delta)$. (Floridi 2011, p. 84 $)^{6}$

There are some potential problems with GDI, but I will not concern myself with them here. ${ }^{7}$ Instead, I focus on one particular quality of semantic information that follows from accepting GDI: the alethic neutrality of semantic information. This is what Floridi

\footnotetext{
2 It is not unreasonable to think that we could have another conception of semantic information, for example, the kind of information that is propositional (perhaps this is more in line with Bar-Hillel's and Carnap's original project).

3 It should be noted that everyone does not agree that a semantic conception of information is of any importance and hence that "there is no need to introduce a concept of semantic information" (Adriaans 2010, cf. Floridi 2010, pp. 259-260).

4 Floridi applies his diaphoric definition of data at three levels: "Data as diaphora de re, that is, as lack of uniformity in the real world out there", "Data as diphora de signo, that is, lack of uniformity between (the perception of) at least two signals", and "Data as diaphora de dicto, that is, lack of uniformity between two symbols" (Floridi 2011, pp. 85-86).

5 It should be noted that all proponents of the veridicality thesis need not accept this. For example, Fred Dretske thinks that "[a]lthough information, as ordinarily understood, may be a semantic concept, this does not mean that we must assimilate it to the concept of meaning" (Dretske 1981, p. 42). Although I agree with Dretske that information is not the same as meaning, in this article, I accept that meaning as a central component of semantic information.

6 It is worth to point out that 'GDI' (general definition of semantic information) has been called other things. For example, in Floridi (2005), 'SDI' (standard definition of semantic information). I will stick with Floridi's later notation, which we also see in Floridi (2016).

7 One problem is that Floridi adds that the meaningful data also needs to be well-formed. However, clearly there are meaningful instances of (semantic) information that are not properly well-formed; an example is this sentence would. This is a problem that goes beyond the philosophy of information but should be of particular interest to that sub-discipline. However, I will not expand on it in this essay. (I have previously discussed this; see Lundgren 2015b.)
} 
questions. He wants to add a fourth criterion to GDI, namely, that meaningful wellformed data also is truthful:

GDI* $\sigma$ is an instance of semantic information if and only if:

GDI*.1 $\sigma$ consists of $n$ data (d), for $n \geq 1$;

$\mathrm{GDI}^{*} .2$ the data are well-formed (wfd);

GDI*.3 the wfd are meaningful $(\operatorname{mwfd}=\delta)$;

GDI*.4 the $\delta$ are truthful (Floridi 2011, p. 104, I have re-named the criteria from 1.-4. to GDI*.1-GDI*.4).

Floridi uses the term 'truthful', rather than true, since, e.g., maps are truthful, but perhaps not true (Floridi 2011, p. 105).

The choice between these two definitions (GDI* or GDI) is not purely technical. We have a conceptual interest in understanding the nature of semantical information, e.g., as a part of our communications. It is in particular as such a concept that an alethically neutral — contrary to a veridical—definition of semantic information (such as GDI) is compatible with our commonsense uses of information. For example, as Pieter Adriaans notes, people tend to accept "If I get the information that $p$ then I know that $p$ " and "Secret services sometimes distribute false information" (Adriaans 2013). What Adriaans' examples show is that the veridical nature of information is ambiguous, which is something that a neutral conception of semantic information can satisfy. These examples illustrate the kind of semantic conception of information that I intend to show can survive Floridi's critique.

\section{A liar paradox for a veridical conception of semantic information}

In this part, I present one basic conceptual problem for a veridical conception of semantic information. Consider a sentence such as (1):

(1) This is not semantic information.

Suppose, which is arguably sensible, that (1) fulfills all necessary and sufficient criteria for semantic information, with the possible exception of (if it is a criterion) veridicality. ${ }^{8}$ Now, if (1) is also true, then (1) fulfills all the criteria of a veridical conception of semantic information and is, accordingly, semantic information, but if (1) is true, then it is also true that (1) is not semantic information. On the other hand, if (1) is false, then (1) does not fulfill all the criteria of a veridical conception of semantic information, and is not, accordingly, semantic information, but if (1) is false, then it is false that (1) is not semantic information, and consequentially, it is true that (1) is semantic information. Therefore, according to a veridical conception of semantic information, (1) is semantic information if, and only if, (1) is not semantic information. I call this the information liar paradox (and sentence 1 is an information liar sentence). It is important to note that the contradiction is a consequence of a veridicality criterion

\footnotetext{
8 If the alternative is between GDI and GDI*, then (1) clearly consists of data, the data in (1) are clearly well-formed, and the well-formed data in (1) are also clearly meaningful.
} 
such as GDI*.4 (under the presumption of, e.g., classical logic), and it is a problem for a veridical conception of semantic information irrespective of any potential solutions to the liar paradox as such (cf. Lundgren (2015a), cf. also Macaulay Ferguson (2015) for a later similar argument).

Proponents of VT have various possible response strategies. I respond to the most plausible strategies in the "Appendix". I argue that even if some response strategies are successful (it is not clear that they are) none will succeed without a cost. Thus, although this argument does not ultimately defeat VT, it is a strong counter-argument that needs to be dealt with.

Let us turn from paradoxical consequences from acceptance of the veridicality thesis to Floridi's first pro-argument for the veridicality thesis and GDI*: the splitting test.

\section{Is false information genuine information?}

Floridi (2005) presents two main arguments in favor of GDI* over GDI. The first, which I will present below (in Sect. 4.1), is a test (the 'splitting test') that is intended to show that false information is not information, which is false, but rather not any kind of information at all. Next, in Sect. 4.2, I argue that the splitting test fails to establish the thesis that false information is not (semantic) information.

\subsection{The 'splitting test'}

The 'splitting test' is based on the Geachian distinction between an attributive adjective and a predicative adjective. The argument is that 'false' in 'false information' is used as an attributive adjective rather than a predicative adjective. Floridi illustrates this distinction with 'male constable' and 'good constable'. In the former, 'male' is a predicative adjective; for a male constable is a "person who is both male and employed as a policeman". In the latter example, we cannot analyze the adjective in terms of a good person who is also employed as a policeman, because 'good' is used as an attributive adjective. Whether an adjective is predicative or attributive can vary with the context (Floridi 2011, p. 97).

Furthermore, we can use attributive adjectives in either a positive or negative way. If used positively, the "adjectives further qualify their reference $x$ as $y$ ", e.g., 'good constable', while if used negatively, the adjective negates "one or more of the qualities necessary for $x$ to be $x$ ". According to Floridi, the negative use is "logically equivalent to "not". An example is 'false constable', in which false indicates that he or she is not a constable at all. Other suggested examples are 'forged banknote', 'counterfeit signature', and 'false alarm' (Floridi 2011, p. 97).

Now, it is suggested that when it comes to false information-i.e., false semantic information - the use of 'false' is negatively attributive:

When we say that $p$, e.g., 'the earth has two moons', is false, we are using 'false' predicatively. The test is that the compound can be split into ' $p$ is a proposition' and ' $p$ is a contingent falsehood' without any semantic loss or 
confusion. On the contrary, when we describe $p$ as false information, we are using 'false' attributively, to negate the fact that $p$ qualifies as information at all. Why? Because 'false information' does not pass the test. As in the case of the false constable, the compound cannot be correctly split. It is not the case, and hence it would be a mistake or an act of misinformation to assert, that $p$ constitutes information about the number of natural satellites orbiting around the earth and is also a falsehood. Compare this case to the one in which we qualify $\sigma$ as digital information, which obviously splits into ' $\sigma$ is information' and ' $\sigma$ is digital'. If false information were a genuine type of information it should pass the splitting test. It does not, so it is not. (Floridi 2011, pp. 97-98).

\subsection{Against the splitting test}

The splitting test may seem methodologically sound, in the sense that if no instances of semantic information pass the splitting test, then that would be a pro-argument for GDI*. However, as Scarantino and Piccinini (2010) argue, the test rests on accepting "the brute intuition that that the earth has two moons is not information. The content of this intuition is nothing but an instance of the general thesis to be established. Thus the argument is question-begging" (p. 321).

The problem, however, is even more severe because the splitting test does not seem to yield the general conclusion that a proponent of VT would desire. As previously noted, Floridi presents several examples of a negatively attributive use of 'false'. Among these examples, the example 'false alarm' seems questionable; for a false alarm is still an alarm. The response to the question "did the alarm go off?" is truly "yes" although it was a false alarm (e.g., a fire alarm gets triggered even though there is no fire). Arguably, a false signature is also still a signature (we could not blame someone for having falsified a signature if he or she had provided no signature at all). ${ }^{9}$

\footnotetext{
9 An anonymous reviewer suggested that "a signature can be defined as such only if the agent executing the act of signing is the owner of the signed name (i.e., what in fact a signature is supposed to be), while a false signature is an imitation". I do not find this counter-example persuasive for two reasons. First, my claim above is based on common-sense agreement of the standard lexical, and (in the case of 'false signature') legal meaning of the word(s). Second, the counter-example fails because it is not necessary for a false signature to be an imitation (it is not even a necessary criterion for success). For example, I could pretend to be someone else and sign documents in that person's name without imitating her signature, simply by using a false ID with a signature that I have made up. That would not constitute an imitation; rather, it would be a case of pretense, yet it would still constitute a false signature (presuming the normal extension of the word). Therefore, imitation cannot be a necessary criterion for a false signature. It could furthermore be argued that $\mathrm{I}$, in fact, own the signature (just as an author is the owner of her pretense). I, of course, recognize that we could make further stipulations that would resolve these issues, but the more we stipulate, the further we get from the standard meaning of the word. One may push the issue further and question what a 'false ID' is, arguing that a false ID requires imitation. I do not think that this helps. First, I do not claim that the principle on which the splitting test rests fails with generality; I claim that the principle fails to hold with generality. Thus, I could grant that a 'false ID' requires imitation and my arguments about the other examples and about false information in particular would still be valid. Second, I believe that thinking that a 'false ID' requires imitation probably depends on an implicit conflation of the false ID and the successful usage thereof. If a kid who does not know what a real ID looks like attempts, but fails, to pass off something as an ID, it may be reasonable to think of that object as a false ID (i.e., it may, as in the example of the signature, be thought of as depending on an act of pretense). Third, even a successful use of a false ID need
} 
Thus, what we should conclude is that the principle on which the splitting test rests does not hold with generality; the examples are ambiguous.

However, it may be argued that even though the examples fail, the test still works for false (semantic) information. Take the example of a witness who gives testimony. Testimony is prima facie semantic information. However, witnesses can lie. Just because a witness lies we would not say that her testimony was not information; rather, we would say that the testimony/information she gave was false (using false in a predicative sense). To support this intuition, think of a situation in which we have two contradictory testimonies. According to a veridical conception of semantic information, a correct description of what the jury must do is to evaluate which of these testimonies is semantic information and which is not. However, this seems contrary to the standard understanding of the concept of information. Furthermore, it seems to be quite counterintuitive: Why should we have to suspend our judgment of whether something is information until we know what is true? Thus, it seems clear that the jury could, would, and should conclude that both testimonies constitute semantic information but that one of these testimonies is false and the other one is true.

The testimony example shows that the use of the concept of information-in colloquial language - tends to vary a lot. As previously mentioned, people tend to accept "If I get the information that $p$ then I know that $p$ " and "Secret services sometimes distribute false information". According to Adriaans (2013), "these inconsistencies do not seem to create great trouble and in general it is clear from the pragmatic context what type of information is designated". It seems clear that when secret services distribute false information their aim is that it should be the kind of information that is essential for epistemic purposes-and clearly, on occasion, they succeed. Because of the ambiguous nature of the concept of information, it seems that the splitting test does not work; in some contexts, it is clear that by 'information' we mean true information, while in other contexts 'information' could be used to indicate false information, overall supporting an alethically neutral conception of information. ${ }^{10}$

It is also worth noting that if we are interested in the kind of information that is essential for epistemic purposes then empirical research shows at the very least "that exposure to negative political information continues to shape attitudes even after the information has been effectively discredited" (Thorson 2016, p. 460). This shows that the role of 'false information' is highly relevant if we are interested in epistemic issues

\footnotetext{
Footnote 9 continued

not require an imitation (even if that probably is common). Two examples come to mind. First, a false ID need not look anything like a real ID (and still be successful). For example, a false ID may be successfully passed off as a genuine ID because it looks like a genuine official document. Now, that may be an imitation of some sort, i.e., of official documents. But my point is not to show that 'false' never functions as it is presumed in the splitting test, but that what is presumed in the splitting test does not hold with generality. Second, a false ID could be a modified real ID (e.g., with a changed picture, information, and signature). The picture, the signature, or the information need not be imitations of any kind, and the rest of the false ID is not an imitation, but the real deal (even if the modification and uses of it invalidate it, making it a false ID). To sum up, what these examples aim to show is that the principle on which the splitting test rests does not hold generally. The main question, which I address in the discussion above, is whether the principle holds generally for 'false' in 'false information'. I conclude that it does not.

10 There are, of course, also contexts that in themselves suppose alethic neutrality, which are supported by the examples previously given.
} 
and that the role of misinformation is, perhaps, not as clear-cut as we philosophers may want it to be. ${ }^{11}$

Let me expand on a few examples that support intuitions contrary to the veridicality thesis and that show, more broadly, that the splitting test does not work. Take, for example, weather forecasts: Is a weather forecast semantic information? Intuitively, this would be a characteristic example of semantic information. But in accordance with the veridicality thesis, we would have to wait and see how reality turns out before we know if we can classify the weather forecast as information. ${ }^{12}$ However, it seems obvious that weather forecasts pass the splitting test. If the weather forecast for tomorrow is that it will be partly sunny and raining three to five millimeters, then it seems intuitive to classify this forecast as semantic information irrespective of how the weather actually turns out. The problem for the splitting test is that our judgment of whether something is information comes before, or at least is separate from, the judgment whether that something is true (truthful) or false. We could imagine examples that are much more extreme, say predictions about the future of the universe 10 million years from now. However, predictions are particularly complicated; therefore, let us turn to other examples. ${ }^{13}$

There are many examples of predictions, statements, and propositions that intuitively seem to be semantic information and would survive the splitting test. Proponents of the veridicality thesis would have to say that Einstein showed that Newton's mechanics is not semantic information. But it is much more reasonable to think that we can classify Newton's mechanics as semantic information and add that it is false. Now, we should note that GDI*.4 actually requires truthfulness, not truth, which may seem to lessen the force of this critique. Maps are strictly false (as their projections presume a flat earth) but still truthful (enough). Likewise, so are Newton's theories-for most applications. However, such a conception of veridical semantic information is, in fact, less convincing. The problem is that what is semantic information would be contextually relative, so while Newton's mechanics would be semantic information in some contexts, it would not be that in other contexts. This is a highly confusing conception of semantic information. ${ }^{14}$ It seems much more sensible to say that Newton's theories are semantic information, information which is strictly not true (but for most purposes sufficiently close to the truth).

\footnotetext{
11 We can, of course, question whether the attitudes Thorson speaks of are the kind of attitudes an epistemologist refers to, i.e., propositional attitudes, but as they affect people's perception of, e.g., a politician, they will play a role in our beliefs of certain propositions, thus playing an indirect, but highly relevant, role in which propositions we accept or deny (granting that belief is necessary for knowledge).

12 Of course, one could say that weather forecasts are always truthful by virtue of being a forecast of the model-based interpretation of the available data, but along those lines, $2+3=4$ could be truthful by virtue of being a report of someone's (distorted) beliefs. But this is not what I am addressing here.

13 Predictions relate to the issue of future contingents, which have been discussed since Aristotle and have gained new interest with the development of temporal logic.

14 It may be that intuitions are split on this example. However, in support of the conclusion above it can be further noted that while the property of being informative can vary under various conditions, whether something is semantic information ought not to be variable, especially not for a proponent of the veridicality thesis. I discuss this in the next section, especially in Sect. 5.2.
} 
Let us turn to Floridi's 'semantic argument', and the final argument for an alethically neutral conception of semantic information.

\section{Must information be informative?}

Floridi's second (2005) argument against a neutral conception of semantic information was merely a sketch at that time, which he developed in Floridi (2007). The argument is rooted in dilemmas concerning informativity and depends partly on the BCP.

These arguments build up to the final conclusive argument in Sect. 5.2, where I show that an alethically neutral conception of semantic information can resolve the $\mathrm{BCP}$, as well as other elements of the semantic argument.

\subsection{BCP and the 'semantic argument'}

Floridi (2005) sketched a semantic argument in one paragraph; the following sentences supply the main locus of the argument:

If false information does not count as semantic junk but as a type of information, it becomes difficult to make sense of the ordinary phenomenon of semantic erosion. Operators like "not" lose their semantic power to corrupt information, information becomes semantically indestructible and the informative content of a repository can decrease only by physical and syntactical manipulation of data. (Floridi 2005, p. 365, cf. 2011, p. 104)

Later, in order to develop this argument, Floridi (2007) introduced 'four principles', which he used to argue that only contingent truths are information. In favor of these principles, Floridi (2011) claimed that "[a]ny satisfactory understanding of semantic information should implement" these principles (p. 98) and that the principles are "uncontroversial and fairly standard assumptions in information theory and in the philosophy of information" (p. 99). However, I will not address the nature of these principles in any great detail, as they are neither argued for nor logically convincing, and contrary to what is claimed, we can question whether they are standard assumptions or not. ${ }^{15}$

15 The principles are not argued for. The claim that the principles are "standard assumptions" (Floridi 2011, p. 99), is done in reference to Bar-Hillel and Carnap (1952, p. 242)*, Dretske (1981), Barwise and Seligman (1997), and van der Lubbe (1997, pp. 10-11) (Floridi 2011, p. 99, fn. 10). We can question the historical accuracy of these interpretation, because, e.g., Bar-Hillel and Carnap distinguish between "[t]he concepts of information and amount of information" (Bar-Hillel and Carnap 1964[1952], p. 221), but even if the sources support Floridi's claim, such support merely constitutes an argument ad populum, or argument ad verecundiam, unless we consider their arguments for the principles.

*Floridi writes 1953, but the year of the original report is 1952. Not to be confused with their 1953 publication in The British Journal for the Philosophy of Science, which covers similar topics.

The principles are logically unconvincing. For example, take: "P. $2 \forall x \forall y((x \neq y) \longrightarrow(H(x \cup y)=$ $H(x)+H(y)))$; additive principle: for any two different instances of information, their overall informative content is equal to the sum of their informative contents", in which " $H(\varphi)=$ primary informative content of $\varphi$ " (Floridi 2011, p. 98). There are three problems. First, it is not clear how we should interpret "different instances of information"; clearly, it does not follow from the formalism. Second, if we have two different 
What is important here is not the four principles as such, because the problem that the semantic argument formulates can be expressed without them. However, one thesis that is necessary for the formulation of this problem is the supposed necessary relation between the property of being informative and the property of being information, which I will question. This problem is exemplified by the fourth principle (P.4), according to which anything that lacks informative content is necessarily not information (ergo, the property of being informative is necessary for the property of being information). This premise is not, as seems to be supposed, obviously true; I question this premise in Sect. 5.2.

The aim of the semantic argument is the conclusion that a conception of semantic information that includes anything but contingent truths is too inclusive. ${ }^{16}$ Two main points of the argument (of relevance for this paper) are as follows.

First, the semantic argument against contradictions (and, arguably, contingent falsehoods) is rooted in the BCP, which is the result that "a self-contradictory sentence, hence one which no ideal receiver would accept, is regarded as carrying with it the most inclusive information" (Bar-Hillel and Carnap 1964[1952], p. 229, quoted in Floridi 2011, p. 100). This result is due to the supposed inverse relation between the informative content (informativity) of some information and its probability (lower probability implies higher informativity). ${ }^{17}$ This basic principle is easily motivated by examples; e.g., if I tell you that we have a meeting next week on Wednesday at 7 p.m., then the truth of this statement is less probable then the truth of the statement that we have a meeting next week. The former is more informative than the latter, because it excludes more alternatives. Based on Bar-Hillel and Carnap's principles (that the informativity of some information is inversely related to its probability) and under the presumption that all information is informative (which Bar-Hillel and Carnap also presume), it can be concluded that:

\section{Footnote 15 continued}

instances of information with a great overlap then their informative content is obviously not equal to the sum of their individual informative content (consider e.g., two books with a partial overlap). In addition, if we apply this rule to three instances of information, it follows that there aren't three independent instances of information with informative content above zero, since from $H(x \cup y \cup z)=H(x \cup y) \cup H(y \cup z)$ it follows that $H(x)+H(y)+H(\mathrm{z})=H(x)+2 H(y)+H(\mathrm{z})$. As pointed out to me by Sven Ove Hansson. Third, the principles are, in fact, incompatible even with GDI*, since according to P.3, tautologies have no informative content, and according to P.4, "any proposition [an instance of what may be information] with no informative content fails to qualify as information" (ibid, p. 99). This latter problem is an easy fix, however.

The principles are not standard assumptions in information theory and in the philosophy of information. An anonymous reviewer pointed out that Kolomogorov complexity, contrary to Floridi's claim above "is nonmonotonous over set theoretical operations", so that "under the assumption that Shannon information and Kolmogorov complexity coincide asymptotically Floridi's principles are far from self-evident for standard non-semantic concepts of information" (see, e.g., Kolmogorov 1965; Shannon and Weaver 1949). The reviewer supplied the following example: Form two sets, A and B, by tossing a coin 1000 times. Number each toss 1-1000, and assign the number to set A if the coin lands heads up, and B if it lands tails up. To characterize the two sets, we would need a random bitstring of 1000 bits, yet their union can be described with only a couple of dozen bits.

16 Again we should note that neither the veridicality thesis nor GDI* excludes tautologies.

17 Bar-Hillel and Carnap supposed a probability space, according to which options were included/excluded by some information, making it less/more probable and more/less informative (see, e.g., Bar-Hillel and Carnap 1964[1952], p. 227). 
Since contradictions are most unlikely, to the point of having zero probability, they are very informative; indeed they are the most informative propositions. (Floridi 2011, p. 100)

The problem is that we rarely take a false statement as very informative, perhaps not informative at all. Proponents of the veridicality thesis have argued that this problem requires a restriction of semantic information which excludes contradictions (Floridi 2011, pp. 100-101; cf. Primiero 2007, p. 406). ${ }^{18}$ The BCP is a problem that an alethically neutral conception of semantic information needs to deal with; I do so in Sect. 5.2.

Second, the semantic argument for the exclusion of contingent falsehoods focuses on the following example: If we accept contingently false information as information, then the informative content of 'Sextus Empiricus died in ad [sic] 201, when Simplicius went to Rome' and 'Sextus Empiricus was alive in ad [sic] 201, when Simplicius went to Alexandria' would be the same. However, "[q]uantitatively, this may be true, but semantically it seems unacceptable. The former would count as information, if true; the latter would not, if false" (Floridi 2011, p. 103). The idea here must be that both sentences are equally probable (given the available information) and therefore, are quantitatively the same. This idea is basically an example of the core of the semantic argument: If false information is information, then "[o]perators like 'not' lose their semantic power to corrupt information" (Floridi 2011, p. 104, cf. quote above). It should be noted, again, that it is evident that the semantic argument takes informativity to be a necessary property for information, which follows from the core of the argument and is directly evident in some of the four principles (such as P.4).

Let us turn to the resolution of these problems.

\subsection{Disentangling informativeness and information}

In this section, I address the importance of distinguishing between what information is from how informative some information is. I do this in order to resolve the BCP (and the rest of the semantic argument), and thus, add to the arguments for an alethically neutral conception of semantic information. However, although my conclusion (which follows from my solution to the BCP and the examples provided in Sect. 4.2) is in favor of an alethically neutral conception of semantic information, I also argue that veridical semantic information is still a meaningful and important subconcept of semantic information.

\footnotetext{
18 The BCP can be established in different ways. Floridi establishes the BCP based on his principles in the following way. He argues that it follows from P.1-P.4 that "if you take any two, different, contingent propositions, then the union of their informative contents is always greater tha[n] the informative content of each of them considered separately" (Floridi 2011, p. 101, corrected 'that' to 'than'). While Floridi argues that it seems problematic that we could increase our informational content by adding contradictions (ibid), it can be questioned if the premise of the argument is even true (see fn. 15, for a critique of the claim that the union of two informative contents is always larger than the informative content of each separately). But as should be clear from the description above, we do not need the four principles to establish the BCP. However, irrespective of how the BCP is established, the informativity of the contradictions leads to the implausible conclusion.
} 
At the core of the semantic argument is the principle that semantic information is necessarily informative. Let us restrict ourselves to that claim. Although it may seem as if the prima facie intuition is on the side of proponents of VT, it is clearly not true on closer analysis. ${ }^{19}$

One may consider some of the examples from Sect. 4.2 directed against the splitting test. If I am right then, e.g., the false statement of a witness constitutes semantic information. Thus, if we follow Floridi in accepting that a false statement is not informative (because it is false), this would be a counter-example to the conception of informativity as a necessary criterion for semantic information, rather than an example of non-information. It is sensible to think that misinformation fails to inform, irrespective of whether misinformation is information or not. If someone provides us with information, and we later find out that it was false, then we may complain, sensibly, that what they told us was not informative. ${ }^{20}$

Now, as previously noted, there is an efficient intuition pump in the semantic argument, i.e., the BCP. The driving force behind the BCP is the idea that if we accept an alethically neutral definition of semantic information then we must accept that contradictions have maximum informational content (which supposedly is counter-intuitive). However, the consequences of the $\mathrm{BCP}$ also depend on the premise that all semantic information is informative. If we avoid conflating the property of being information with the property of being informative, then there is no paradox to speak of. The technical issue, which follows if we want to retain the operators used by Bar-Hillel and Carnap, can be fixed, perhaps in the way suggested by Floridi (2004, 2011, chapter 5 ); but the philosophical issue is resolved by realizing that there was no problem to begin with. We can have an alethically neutral definition of semantic information and, if we want, still claim that the informativity of semantic information depends on truth. Thus, we can deal with the question of informativity as a concept relating to truth without needing to accept any version of the veridicality thesis. ${ }^{21}$ The same argument is applicable to contingently false information; i.e., that false information generally is less informative than truthful information (or not informative at all as the example of the incorrect changes in the historical accounts of Sextus Empiricus aims to

\footnotetext{
19 Two things are worth noting. First, that the presumption that all information is informative is not only held by proponents of VT. Second, while Floridi's argumentation generally assumes that what is not informative is not information (cf. also Floridi 2011, pp. 94-96), he does, at one moment, recognize an essential distinction: He states that "following P.1 [the principle that no information can have negative informativity] and P.4, they [tautologies] may be represented as instances of information devoid of any informativeness" (Floridi 2011, p. 99, my addition within brackets, cf. Floridi 2011, p. 98). This is an exception to the rest of Floridi's argumentation, and it is incompatible with P. 4.

20 It may be argued that informativity requires more than that the information is truthful; e.g., in colloquial language we usually speak of truthful information as not being informative because it was not that particular information that we wanted or needed in that specific context, or because we already knew it. A related example would be to claim that something is less informative because we can generally expect it to be more well-known, e.g., that the chemical formula of water is $\mathrm{H}_{2} \mathrm{O}$ may be considered less informative than that the chemical formula of hydrogen peroxide is $\mathrm{H}_{2} \mathrm{O}_{2}$, ceteris paribus.

21 We also need to settle the issue of whether tautologies are informative or not, but this is an issue that can be coherently resolved with an alethically neutral conception of semantic information while it is more problematic for a veridical conception of semantic information, which conflates the property of being informative with the property of being information.
} 
show) does not mean that false information is not information. It just says something about the informativity of false information. Likewise, it is much more reasonable to think that Newton's theories are information, but their informativeness varies with the requirements of various applications.

BCP served as part of the basis for the dilemma that Macaulay Ferguson had in mind when he argued that we have to choose between two paradoxes. On the one hand, we have the theories of Bar-Hillel and Carnap, which allow false information as semantic information and therefore suffer from the BCP, and on the other hand, we have the theories of Fred Dretske and Luciano Floridi, which suffer from counter-examples such as the information liar sentence (Macaulay Ferguson 2015). This dilemma is resolved by giving up the conflation of informativeness with (semantic) information. We can then accept some alethically neutral definition of semantic information while dealing with informativeness as a separate issue.

The core of the semantic argument was that "operators like 'not' lose their semantic power to corrupt information" (see Floridi 2005, p. 365, 2011, p. 104). Now, it is true that 'not' (when used in a meaningful sense) cannot change semantic information either into non-semantic information or into non-information. However, from this it does not follow that operators like 'not' lack semantic power to corrupt information, because they can still be used to make some informative semantic information noninformative (and vice versa).

Thus, disentangling the property of being information and the property of being informative resolves the semantic argument in favor of an alethically neutral conception of semantic information. Although this insight is generally compatible with various other conceptualizations of the two concepts, and informativity, in particular, a few properties follow as a corollary. The main difference between the property of being information and the property of being informative is that the latter takes on part of the properties that information supposedly has under the veridicality thesis. Under VT, whether some data are information is contingent and depends on the truth of that information, e.g., on the state of the world. In Sect. 4.2, I supply various examples that aim to show that this idea is false. ${ }^{22}$ Thus, whether some data constitute information does not depend, e.g., on the state of the world. However, whether something is informative arguably does. ${ }^{23}$ This is why I have argued that we ought to retain veridical semantic information as a subconcept of semantic information.

Finally, much more could be said about the properties of these concepts. However, as I wish the main insight of this article to remain as compatible with as many accounts of informativity as possible I will not make any suggestions for further qualifications of the two concepts here.

\footnotetext{
22 For example, we need not wait to see if future predictions come true in order to determine whether they constitute information, but we may need to wait to see whether they were, in fact, informative. Nor do we need to disqualify theories of science as not information when errors are uncovered; however, after the facts are clear, we can revise the status of how informative it is.

23 Arguably, whether something is informative for a specific epistemic agent also depends on other criteria, cf. fn. 20.
} 


\section{Conclusions}

In this article, I have argued that a veridical conception of semantic information suffers from contradictions such that some sentence is information if, and only if, it is not information. Furthermore, I have argued against the splitting test, which aims to show that false information is not genuine information, and I have presented counter-examples against a veridical conception of semantic information. Last, based on a distinction between the property of being informative and the property of being information (supported partly based the intuition-driven arguments) I resolved some semantic arguments, such as the BCP, directed against an alethically neutral conception of semantic information, while re-introducing veridical semantic information as a subconcept of semantic information.

In summary, I hope to have shown that there are good reasons to adopt a nonveridical conception of semantic information. I also hope to have shown that veridical semantic information is still useful, as a subconcept of semantic information.

Acknowledgements Many thanks to three anonymous reviewers for Synthese, as well as John Cantwell, Sven Ove Hansson, Niklas Möller, Fredrik Stjernberg, and Tor Sandqvist for their excellent comments. Furthermore, I want to thank the attendees for their comments when I presented some of the ideas in this paper and/or earlier drafts at the Swedish Philosophy Conference, at the higher seminars in philosophy at the Royal Institute of Technology, and at the defense of my licentiate thesis. Finally, I want to thank the Swedish Civil Contingency Agency for providing funding that enabled me to write this article.

Funding The author ‘s employment is funded by MSB (Swedish Civil Contingencies Agency) for research performed in a project concerning Information Security Culture.

Open Access This article is distributed under the terms of the Creative Commons Attribution 4.0 International License (http://creativecommons.org/licenses/by/4.0/), which permits unrestricted use, distribution, and reproduction in any medium, provided you give appropriate credit to the original author(s) and the source, provide a link to the Creative Commons license, and indicate if changes were made.

\section{Appendix: Further arguments on the information liar paradox}

Let us briefly re-iterate the information liar paradox. Consider a sentence such as (1):

(1) This is not semantic information.

If (1) is also true, then it is also true that (1) is not semantic information. If (1) is false, then (1) does not fulfill all the criteria of a veridical conception of semantic information, and is not, accordingly, semantic information, but yet, if (1) is false, then it is false that (1) is not semantic information, and consequentially, it is true that (1) is semantic information. Therefore, according to a veridical conception of semantic information (1) is semantic information if, and only if, (1) is not semantic information. This paradox holds only under the presumption of VT. Below, I consider four objection strategies for proponents of VT. I argue that although this argument does not ultimately defeat VT, a proponent of VT cannot defend against it without a cost. Finally, I also consider a twist to the paradox (concerning the concept of veridical semantic information). 
A first objection strategy is to object that (1) is, in fact, a logical necessity. ${ }^{24}$ Although a strict proponent of VT, as usually formulated, should accept tautologies as information, not all of them do, which would potentially resolve the paradox. For example, although tautologies are information according to GDI*, Floridi thinks that tautologies fail to be information because they are not informative (Floridi 2011, p. 99). ${ }^{25}$ However, the following sentence, $(1 *)$, is arguably neither a logical necessity (nor a tautology), while it retains the problem discussed above: The sentence written at the end of this paragraph is not semantic information.

A second objection strategy is to object that (1) and (1*) fail to be semantic information due to criteria other than veridicality, e.g., that they are not meaningful. In order for this objection to be successful, we need to understand that under AN (1) and ( $\left.1^{*}\right)$ can unproblematically be classified as simply false, though meaningful. More importantly, we also have good reason to think that (1) and $\left(1^{*}\right)$ are meaningful. There are at least two reasons for considering (1) and $(1 *)$ as meaningful.

First, they are commonsensically meaningful, since they are perfectly understandable, communicable, and without any theoretical pre-conceptions (such as VT) unproblematic.

Second, sentences (1) and (1*) are quite similar to other, false, self-referential sentences that we would consider meaningful, such as 'This sentence consists of seven words.' There is a multitude of such (false) self-referential sentences, and classifying them as not meaningful is prima facie unconvincing.

Thus, an objection that (1) and (1*) are not meaningful needs to be supported by a stronger conception of the criterion of meaningfulness than the one presumed in this article. There may be arguments for such a conception, but if the only reason we have for claiming that (1) and (1*) are not meaningful is based on a belief in VT, then that is not a very good reason-it is circular at best. Without a specific and, in the relevant sense, non-circular argument in favor of a conception of meaningfulness that excludes (1) and (1*), the claim that (1) and (1*) are not meaningful is merely an ad-hoc solution, by proof-by-assertion, to a problem for VT. It is questionable whether such arguments are forthcoming. I will briefly consider two possible argumentative strategies below.

First, sentences like (1) and $\left(1^{*}\right)$ are expressible only in very rich language; specifically, sentence (1) requires a language with indexicals. One may worry that the meaningfulness of $(1)$ and $(1 *)$ is contextually relative. However, AN offers a language that is at least as rich as that offered under the presumption of VT; still, (1) and $(1 *)$ are paradoxical under VT, but not under AN. Therefore, the richness of the language cannot be an issue. Furthermore, the paradox can be established by sentences that do not depend on indexicals. For example, let us assign the sentence below the name ' $(1 * *)$ ', i.e., the sentence that follows after ' $(1 * *)$ ':

$\left(1^{* *}\right)$ Sentence $\left(1^{* *}\right)$ is not information.

\footnotetext{
24 Thanks to Fredrik Stjernberg for this argument.

25 Interestingly, Floridi (2011) notes that tautologies "may be represented as instances of information devoid of any informativeness" (p. 99). This, however, is not an idea he further pursues.
} 
As before, sentence $\left(1^{* *}\right)$ yields a paradox under the presumption of VT, but not under AN. ${ }^{26}$

Second, proponents of VT can argue that meaning requires us to be able to consistently assign truth-values to the information (i.e., a sentence is meaningful only if it can be assigned a truth-value). Again, remember that $(1),\left(1^{*}\right)$, and $\left(1^{* *}\right)$ can be assigned truth-values under AN. ${ }^{27}$ Thus, (1), (1*), and (1**) lack meaning only under the presumption that VT is true, which means that such an argument begs the question.

There may be other possible arguments for the meaningless of $(1),\left(1^{*}\right)$, and $\left(1^{* *}\right)$ that avoids begging the question. However, as I will argue, no such argument can completely resolve the issue, because we can always construct an information liar revenge sentence as follows:

(2) This sentence is either not information and meaningful, or else it is not meaningful.

If (2) is false, then it has to be meaningful (otherwise, it is true, because of the second disjunct), and it has to be information (otherwise, it is true, because of the first disjunct). So, if (2) is false, then (2) is false, meaningful, and information, which conflicts with VT, but not AN. So, according to VT, (2) cannot be false. Proponents of VT may now suggest that (2) is not meaningful. However, if (2) is not meaningful, then it must be true, as it follows that the second disjunct is true (and because of this, the first disjunct is false). ${ }^{28}$ Thus, if (2) is not meaningful, then (2) is true, which is, arguably, problematic. Therefore, (2) has to be true, which means that (2) is either not information, but meaningful and true (which cannot be the case according to VT) or (2) is true, but not meaningful. To sum up, under VT, (2) cannot be false, but we have to conclude that it is true and not meaningful.

Unlike the liar's revenge (cf. Beall 2007), this revenge problem does not necessarily end up in a paradox, i.e., if a proponent of VT requires that information be meaningful and true. However, it is still problematic and plausible unacceptable, because proponents of VT would have to argue that sentences such as (1), $(1 *),\left(1^{* *}\right)$, and (2) fail to be semantic information because they fail to be meaningful, but at the same time accept that truth does not depend on meaning, i.e., that there are true sentences, which are not meaningful (and, of course, because of this, not information). ${ }^{29}$ There are at least two problems. First, it is questionable if a proponent of VT would accept this (for

\footnotetext{
26 Of course, it could be claimed that ( $\left.1^{* *}\right)$, similar to (1), is a logical necessity. But the point here is that indexicals cannot be the source of the problem.

27 Note, also, that many philosophers think that we can in fact assign truth-values to liar sentences either in paracomplete or paraconsistent logics (see, e.g., Kripke 1975; Beall 2009; Priest 2006). Restricting the requirement that some $\mathrm{x}$ is only meaningful if it can be assigned a truth-value that is either true or false does not help either, since under AN (1) and ( $\left.1^{*}\right)$ can be assigned such a truth-value.

28 The first disjunct is a conjunction which states (2) is not information and (2) is meaningful. If (2) is not meaningful, then the second conjunct is false. (Also, if (2) is not meaningful and we presume that if $\mathrm{x}$ is not meaningful it is not information, then the first conjunct must be true.) So, the first disjunct must be false.

29 Although some have questioned the semantic role of meaning (cf. Dretske 1981, p. 42, quoted in fn. 5 , p. 5), it does not resolve the issue, since if one denies meaning as a criterion for information then we have no reason to deny that $(1),(1 *)$, or (2) are semantic information. Thus, such suggestions are a no go for the proponents of VT.
} 
example, a proponent of GDI* likely would not). ${ }^{30}$ Second, even if someone would accept it, it is questionable if he or she can produce a convincing argument for it, an argument which does not beg the question. Either way, the paradox cannot be resolved without a substantial cost.

A third objection strategy is to argue that a sentence like (1), $\left(1^{*}\right),\left(1^{* *}\right)$, or (2) does not qualify as information that is essential for epistemic purposes, because it is paradoxical. However, such an objection fails for two reasons. First, it depends on the presumption that the sentence is paradoxical, which begs the question. More importantly, this objection is arguably also wrong, since there is nothing paradoxical about the sentence in itself. It can consistently be assigned a truth-value, and the sentence provides a paradox only under the presumption of VT. This means that it is up to the proponent of VT to argue that the paradox is a problem due to the sentence, not VT. Second, it is also wrong to think that sentences that yield paradoxes under other presumptions are not essential for epistemic purposes. In fact, many paradoxes are essential for epistemic purposes; think, e.g., of the role of Russell's paradox. ${ }^{31}$

The problem could be further explicated by arguing that, unlike the device used by Russell, sentences like (1), (1*), (1**), or (2) do not have any further epistemic function beyond what we can learn by considering this paradox. Thus, one may argue that the epistemic criterion that proponents of VT are after is not about meta-theoretical properties, but about properties external to the sentences under consideration. However, I think that the sentences under consideration qualify as epistemically useful beyond this example. The device that I make use of is sentences-structures such as ' $x$ is (not) information', a fundamental sentence schema. This schema is used in the paradox to show that the presumption of VT yields a paradox. In its purest form, we may apply it as follows:

(y) Sentence $\mathrm{x}$ is not information.

If sentence $\mathrm{x}$ is ' $3+4=5$ ', then (y) is true under the presumption of VT, but false under AN. This shows that the sentence schema can be used to exemplify the difference between VT and AN, without reference to the paradox. Thus, the sentence schema is useful beyond the meta-theoretical considerations that I have focused on in this "Appendix". The schema works in all cases under AN, and most cases under VT, and it is (as I just shown) epistemically useful beyond the paradox. We can also use the exact same schema to yield the paradox. If sentence $(\mathrm{x})$ reads as follows:

(x) Sentence y is information.

Then, under the presumption of VT, we have a paradox. Thus, the basic sentence schema, which is used in order to construct the paradox, is a standard device that is used regularly — and without any problem—when we evaluate sentences.

A fourth objection strategy is to attempt to argue for restrictions of the language so that sentences like $(1),(1 *),(1 * *)$, and (2) are not part of the language, thus avoiding

\footnotetext{
30 GDI* indicates a structure in which truthfulness depends on meaning (and meaning depends on wellformedness, and that all this applies to data). See p. 5.

31 Thanks to an anonymous reviewer for this example. Cf. fn. 34, p. 26 for a related discussion.
} 
to yield a paradox under VT. For example, one may consider if a Tarskian solution is satisfactory, i.e., by recognizing that what is true or information in our colloquial (object) language can be expressed only in a meta-language (cf., e.g., Tarski 1944; 1983). There are several problems with such an approach.

First, a proponent of VT would have to argue that the Yabloian modification of the information liar paradox does not work (Yablo 1993). But such a paradox could easily be created. ${ }^{32}$ However, we have to acknowledge that the Yabloian modification of the information liar paradox might be less of a problem because it has been contested whether Yablo's paradox lacks self-reference and thus that it can, in fact, be technically resolved by Tarskian requirements (see, e.g., Priest 1997; Beall 2001, cf., e.g., Sorensen 1998 for a defense of the non-circularity of Yablo's paradox).

Second, Tarski intended his solution for formal languages. He questioned "the very possibility of a consistent use of the expression 'true sentence' which is in harmony with the laws of logic and the spirit of everyday language" (Tarski 1983, p. 165). But the concept of semantic information is supposed to be applicable to colloquial language. Thus, it is questionable if the restrictions required to solve this problem are something that we should accept. If we did, then many sentences that ordinarily would be information, according to VT and AN, would fail to be information just because they involve the predicate is true or is information. In fact, the veridicality thesis would not be expressible in the object language. Thus, although this is possibly a plausible solution, it cannot be achieved without a cost. Arguably, this cost is too severe.

Third, even if we could accept such restrictions, what reason do we have for doing so? A possible motivation would be to say that if we accept the T-schema, we accept that: If ' $p$ ' is true, then $p$. Similarly, proponents of VT think that we should accept: If ' $p$ ' is semantic information, then $p$. However, the similarities of the cases are also, in fact, illusionary. Although we have an enormous literature on the liar paradox, specifically, and truth, in general, that could support blocking the liar paradox (and similar devices for solving other well-known problems with truth) we are not in a similar situation in relation to the concept of semantic information. ${ }^{33}$ Thus, although a technical solution may have been possible to motivate for a problem that directly involves truth, this suggestion is less convincing for the current problem. Instead, this needs to be regarded as a genuine problem for VT. Furthermore, it may be argued that this motivation (If ' $p$ ' is semantic information, then $p$.) is, in fact, what we are arguing about, thus making it question-begging (as a comparison, in the case of the

\footnotetext{
32 Take an infinite sequence of sentences such that:

- (S1) For each $\mathrm{i}>1, \mathrm{Si}$ is not information.

- (S2) For each $\mathrm{i}>2$, Si is not information.

- ...
}

If any sentence $S_{n}$ is true, then every sentence $S_{>n}$ is not information, but then every sentence $S_{>n}$ is true and according to VT, information. But then it follows that there is some information which is not information. On the other hand, if any sentence $\mathrm{S}_{n}$ is false, then some sentence $\mathrm{S}_{>n}$ is information (let us denote such a sentence ' $S_{x}$ '). Given that $S_{x}$ is information, it follows, according to VT, that it must be true, but then every sentence $\mathrm{S}_{>x}$ is not information, and thus, every sentence $\mathrm{S}_{>x}$ is true and according to VT, information. But then it follows that there is some information which is not information.

33 Thanks to John Cantwell for presenting this problem and to Fredrik Stjernberg for suggesting this response. 
liar paradox, we are not usually arguing about the truth of the T-schema). If all a proponent of VT can say is that we need to accept these restrictions in order to resolve the information liar paradox, then such reasons beg the question.

Although I do not think that this discussion is the end of this topic, or that paradox is ultimately decisive on the issue of VT versus AN, it is nevertheless something that a proponent of VT needs to deal with. In addition, if I am right, it is something that proponents of VT cannot deal with without a cost.

Last, in Sect. 5 I introduced the concept of veridical semantic information, arguing that it is essential for the analysis of informativity. One may worry that veridical semantic information suffers from paradox. For example, consider the following sentence: 'This sentence is not veridical semantic information.' However, this is less worrisome than the problem discussed above.

First, because this formulation depends on truth ('veridicality') it can consistently be argued that this is the kind of problem that truth suffers from. Thus, it is reasonable to argue that - unlike the information liar sentences - this is just a re-formulation of the liar sentence. For this reason, and given that we are dealing with a term of art, we can motivate various simple solutions. For example, a classical logician may note that truth requires consistency, ignoring the sentence. This would be supported by the $\mathrm{BCP}$, which depends on the intuitive argument that inconsistencies are, in fact, not informative, ${ }^{34}$ while a paraconsistent logician may think that the sentence poses no problem whatsoever.

Second, my argument for retaining the subconcept also serves as a potential way to resolve the paradox: If proponents of VT and AN can agree that informativity depends on truth, then such paradoxes can be satisfactorily resolved with technical solutions. ${ }^{35}$ My point here is that the dialectics have changed: If we have already agreed that veridicality (in some formulation) is a necessary criterion for informativity, then it would not beg the question to resolve any issues related to such a concept by presuming what is already in evidence. I recognize, of course, that there may still be proponents of contrary positions that would think that issue is not yet resolved. ${ }^{36}$ If so, there may be a debate to be had, but the debate would be about the property of being informative, rather than the property of being information.

\footnotetext{
${ }^{34}$ Remember that the BCP and the concept are related to informativity. Note also that this may seem to contradict my previous argument that a sentence that yields a paradox under other presumptions can be essential for epistemic purposes. However, this does not mean that the actual sentences are informative in themselves about themselves. Rather, if they are informative, they are informative in the sense that, e.g., Russell's paradox was informative about the inconsistency in naïve set-theory, or because the sentence schema can be used for other epistemic purposes. The result of a paradox, thus, can be informative. However, this is a complex issue that I will not expand upon further.

35 Needless to say, an agreement that informativity depends on truth does not mean that concept of informativity cannot be spelled out in very many competing ways, even in relation to truth.

36 For example, proponents of the original Bar-Hillel and Carnap approach, in which truth is not a requirement for informativity.
} 


\section{References}

Adriaans, P. (2010). A critical analysis of Floridi's theory of semantic information. Knowledge, Technology \& Policy, 23, 41-56.

Adriaans, P. (2013) . Information. In E. N. Zalta (Ed.), The stanford encyclopedia of philosophy (Fall 2013 Edition). http://plato.stanford.edu/archives/fall2013/entries/information/

Bar-Hillel, Y., \& Carnap, R. (1953). Semantic information. The British Journal for the Philosophy of Science, 4(14), 147-157.

Bar-Hillel, Y., \& Carnap, R. (1964[1952]). An outline of a theory of semantic information. In Y. Bar-Hillel (1964) (Ed.), Language and information: Selected essays on their theory and application. London.

Barwise, J., \& Seligman, J. (1997). Information flow: The logic of distributed systems. New York: Cambridge University Press.

Beall, J. C. (2001). Is Yablo's paradox non-circular? Analysis, 61(3), 176-187.

Beall, J. C. (2007). Prolegomenon to future revenge. In J. C. Beall (Ed.), Revenge of the Liar: New essays on the paradox. Oxford: Oxford University Press.

Beall, J. C. (2009). Spandrels of truth. Oxford: Clarendon Press.

Dodig-Crnkovic, G. (2005). System modeling and information semantics. In J. Bubenko, et al. (Eds.), Promote IT 2005: Proceedings of the fifth conference for the promotion of research in IT at New Universities and University Colleges in Sweden: Borlänge, Sweden 11-13 May, 2005 (pp. 119-128). Lund: Studentlitteratur.

Dretske, F. I. (1981). Knowledge and flow of information. Oxford: Blackwell (also available in a more recent printing. 1999. Stanford, CA: CSLI Publications)

Fetzer, J. H. (2004). Information: Does it have to be true? Minds and Machines, 14(2), 223-229.

Floridi, L. (2004). Outline of a theory of strongly semantic information. Minds and Machines, 14(2), 197222.

Floridi, L. (2005). Is semantic information meaningful data? Philosophy and Phenomenological Research, $70,351-70$.

Floridi, L. (2007). In defence of the verdidical nature of semantic information. EUJAP, 3(1), 31-41.

Floridi, L. (2010). The philosophy of information as a conceptual framework. Knowledge, Technology \& Policy, 23, 253-281.

Floridi, L. (2011). The philosophy of information. Oxford: Oxford University Press.

Floridi, L. (2012). Semantic information and the network theory. Synthese, 184, 431-54.

Floridi, L. (2016). Semantic conceptions of information. In E. N. Zalta (Ed.), The stanford encyclopedia of philosophy (Spring 2016 Edition). https://plato.stanford.edu/entries/information-semantic/.

Fox, C. J. (1983). Information and misinformation: An investigation of the notions of information, misinformation, informing, and misinforming. Westport, CT: Greenwood P.

Graham, G. (1999). The Internet: A philosophical inquiry. London: Routledge.

Grice, H. P. (1989). Studies in the way of words. Cambridge, MA: Harvard University Press.

Kripke, S. (1975). Outline of a theory of truth. Journal of Philosophy, 72(19): 690-716. Reprinted in Kripke, S. A. (2011). Philosophical troubles: Collected papers (Vol. 1). New York: Oxford University Press.

Kolmogorov, A. N. (1965). Three approaches to the quantitative definition of information. Problems of Information Transmission, 1(1), 1-7.

Macaulay Ferguson, T. (2015). Two paradoxes of semantic information. Synthese, 192(11), 3719-3730.

Lundgren, B. (2015a). The information liar paradox: A problem for Floridi's RSDI definition. Philosophy and Technology, 28(2), 323-327.

Lundgren, B. (2015b). Why semantic information is only meaningful data. Presentation at the Swedish Philosophy Conference. Linköping University.

Priest, G. (1997). Yablo's paradox. Analysis, 57(4), 236-242.

Priest, G. (2006). Doubt truth to be a liar. Oxford: Oxford University Press.

Primiero, G. (2007). An epistemic constructive definition of information. Logique \& Analyse, 200, 391-416.

Scarantino, A., \& Piccinini, G. (2010). Information without truth. Metaphilosophy, 41(3), 313-330.

Sorensen, R. (1998). Yablo's paradox and kindred infinite liars. Mind, 107(425), 137-155.

Shannon, C. E., \& Weaver, W. (1949). The mathematical theory of communication. Urbana, IL: University of Illinois Press (also available in a more recent printing. 1998. Urbana: University of Illinois Press)

Tarski, A. (1944). The semantic concept of truth: And the foundations of semantics. Philosophy and Phenomenological Research, 4(3), 341-376. 
Tarski, A. (1983). Concept of truth in formalized languages. In Logic, semantics, metamathematics: papers from 1923 to 1983 (2nd edn). Indianapolis: Hackett Publishing Company.

Thorson, E. (2016). Belief echoes: The persistent effects of corrected misinformation. Political Communication, 33, 460-480.

Yablo, S. (1993). Paradox without self-reference. Analysis, 53(4), 251-252. 